\title{
Entrapment ability and release profile of corticosteroids from starch-based microparticles
}

\author{
G. A. Silva, ${ }^{1,2}$ F. J. Costa, ${ }^{1}$ N. M. Neves, ${ }^{1,2}$ O. P. Coutinho, ${ }^{1,3}$ A. C. P. Dias, ${ }^{3}$ R. L. Reis ${ }^{1,2}$ \\ ${ }^{1} 3 B^{\prime}$ s Research Group - Biomaterials, Biodegradables, Biomimetics, University of Minho, Campus de Gualtar, 4710-057, \\ Braga, Portugal \\ ${ }^{2}$ Department of Polymer Engineering, University of Minho, Campus de Azurém, 4800-058 Guimarães, Portugal \\ ${ }^{3}$ Department of Biology, University of Minho, Campus de Gualtar, 4710-057 Braga, Portugal
}

Received 13 September 2004; revised 2 November 2004; accepted 19 November 2004

Published online 10 March 2005 in Wiley InterScience (www.interscience.wiley.com). DOI: 10.1002/jbm.a.30287

\begin{abstract}
We previously described the synthesis of starchbased microparticles that were shown to be bioactive (when combined with Bioactive Glass 45S5) and noncytotoxic. To further assess their potential for biomedical applications such as controlled release, three corticosteroids with a similar basic structure-dexamethasone (DEX), 16 $\alpha$-methylprednisonole (MP), and 16 $\alpha$-methylprednisolone acetate (MPA) - were used as models for the entrapment and release of bioactive agents. DEX, MP, and MPA were entrapped into starch-based microparticles at $10 \% \mathrm{wt} / \mathrm{wt}$ of the starch-based polymer and the loading efficiencies, as well as the release profiles, were evaluated. Differences were found for the loading efficiencies of the three corticosteroids, with DEX and MPA being the most successfully loaded (82 and $84 \%$, respectively), followed by MP (51\%). These differences might be explained based on the differential distribution of the molecules within the matrix of the microparticles. Furthermore, a differential burst release was observed in the first $24 \mathrm{~h}$ for all corticosteroids with DEX and MP being
\end{abstract}

more pronounced (around 25\%), whereas only 12\% of MPA was released during the same time period. Whereas the water uptake profile can account for this first stage burst release, the subsequent slower release stage was mainly attributed to degradation of the microparticle network. Differences in the release profiles can be explained based on the structure of the molecule, because MPA, a more bulky and hydrophobic molecule, is released at a slower rate compared with DEX and MP. In this work, it is shown that these carriers were able to sustain a controlled release of the entrapped corticosteroids over 30 days, which confirms the potential of these systems to be used as carriers for the delivery of bioactive agents. (C) 2005 Wiley Periodicals, Inc. J Biomed Mater Res 73A: 234-243, 2005

Key words: biodegradable polymers; starch-based microparticles; drug release; dexamethasone; methylprednisolone; biomaterials

\section{INTRODUCTION}

Because of the considerable advantage of their clearance from the body after the release of therapeutic agents, biodegradable polymers are among the most widely used materials for controlled drug delivery applications. ${ }^{1}$ Starch-based polymers have been studied mainly by Reis et $\mathrm{al}^{2-5}$ for a wide range of bonerelated applications, ranging from tissue engineering scaffolds, ${ }^{6-11}$ to bone cements, ${ }^{12-14}$ and drug delivery

Correspondence to: G. A. Silva; e-mail: gsilva@dep. uminho.pt.

Contract grant sponsor: Portuguese Foundation for Science and Technology; contract grant number: SFRH/BD/ $4648 / 2001$

Contract grant sponsor: Portuguese Foundation for Science and Technology through funds from the POCTI and/or FEDER programs

(C) 2005 Wiley Periodicals, Inc. systems. ${ }^{14-16}$ These materials display a set of features that support their potential in the biomedical field, such as natural origin, good mechanical properties, ${ }^{3,11,17-19}$ good biological performance, ${ }^{20-25}$ and the possibility of tailoring their properties ${ }^{26-29}$ according to the foreseen application. Recently, we have described the use of a blend of starch and polylactic acid (SPLA50) to synthesize microparticles (polymer and composite with Bioactive Glass 45S5) with a defined size range that were found to be bioactive and noncytotoxic. $^{30}$ A potential application for these developed microparticles is as carriers for bioactive agents in controlled release applications.

There are two basic general strategies to develop polymeric matrices for delivery systems: the use of hydrophobic matrices that can release encapsulated drugs as a result of their bioerosion or biodegradation, or the use of hydrogel matrices, that can swell and retain large volumes of water, thus allowing diffusion 
of the drug. ${ }^{31}$ The combination of these two strategies (by using a blend of hydrophobic-hydrophilic polymers, such as polylactic acid and starch) might allow for the synthesis of a carrier that combines the best features of each material. Factors influencing release rate include drug molecular size and loading efficiency into the polymer, polymer composition and molecular weight, and the dimensions, shape, and crystallinity of the matrix. ${ }^{31,32}$

Considering all these aspects, the entrapment of bioactive molecules within starch-based microparticles and subsequent release profile were evaluated to assess the potential of these micron-size systems to be used as carriers for drug delivery. For this purpose, three different corticosteroids were chosen as model bioactive agents. Corticosteroids have a widespread use in clinical practice because of their broad range of antiinflammatory activities; they are used in affections such as acute respiratory distress, degenerative diseases, as well as immunosuppressors in organ transplantation. ${ }^{33}$ Dexamethasone (DEX) has a high estimated potency, ${ }^{34}$ being used for several applications, in which ocular ones have a major importance. DEX delivery systems for eye delivery, as well as for preventing stent restenosis, have been documented. ${ }^{34-36}$ It has also been widely used in in vitro cell culture where differentiation of bone marrow cells into the osteoblastic lineage is to be achieved. ${ }^{37}$ Methylprednisolone is used similarly to DEX, finding applications in endocrine and rheumatic disorders as adjunctive therapy for short-term administration, collagen diseases during exacerbation or as maintenance therapy, and also in several dermatological diseases (psoriasis, dermatitis, Stevens-Johnson syndrome).

In the present work, we describe the ability of starch-based microparticles to act as carriers for drug delivery applications, by means of entrapping and evaluating the release profiles of three corticosteroids,

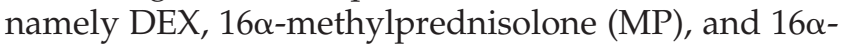
methylprednisolone acetate (MPA).

\section{EXPERIMENTAL}

\section{Materials}

A blend of starch and polylactic acid (PLA) herein referred to as SPLA50 (50\% cornstarch, 50\% PLA, by weight) was used as the raw material. The PLA used in this blend was PLA 4040, 94\% L-lactide from Cargill-Dow (Minneapolis, MN), and the blended material was supplied by Novamont (Novara, Italy). More information on this material can be found elsewhere. ${ }^{7}$ Methylene chloride (Sigma, St Louis, MO) and polyvinyl alcohol 87-89\% hydrolyzed (Aldrich, Milwaukee, WI) were used for the production of the microparticles. DEX $\geq 98 \%$ [high-performance liquid chromatog- raphy (HPLC) grade; Sigma], $\mathrm{MP} \geq 99 \%$, and $\mathrm{MPA} \geq 99.3 \%$ (both in-house produced) were used as the bioactive molecules for entrapment and release studies. All other chemicals used were reagent grade (Panreac, Barcelona, Spain) except methanol, which was HPLC grade (Riedel-de-Haën, Sigma/ Aldrich, Seetze, Germany).

\section{Synthesis of starch-based microparticles loaded with corticosteroids}

The synthesis methodology for SPLA50 microparticles was as described previously. ${ }^{30}$ Briefly, SPLA50 was mixed with the steroid (10\% weight of polymer) and dissolved in methylene chloride. This solution was then emulsified with a stirring $0.5 \%$ polyvinyl alcohol solution for up to $4 \mathrm{~h}$ in a top stirrer at a speed of $660 \mathrm{rpm} / \mathrm{min}$. The microparticles were then washed and separated from the reaction solution by filtration. The reaction solution was stored for quantification of the nonloaded corticosteroid. Steroid-loaded microparticles were freeze-dried and stored in a desiccator until further use. This procedure was performed separately for each of the corticosteroids used, namely, DEX, MP, and MPA. Values of 2.5, 5, and 10\% wt/wt (DEX/polymer) were tested to determine the ratio of bioactive agent to polymer that achieved the highest encapsulation efficiency. At least three independent experiments were performed for each of the corticosteroids with reproducible results.

\section{Physicochemical characterization of SPLA50 microparticles}

Starch-based microparticles-unloaded, steroid-loaded, and after a 30-day release period-were gold coated and analyzed by scanning electron microscopy using a Leica Cambridge S-360 model (Cambridge, UK) to assess morphological features and differences between conditions.

To assess changes in the microparticles between pre- and postrelease, Fourier transformed infrared attenuated reflection (FTIR-ATR) was performed. Spectra were recorded at least at 32 scans with a resolution of $2 \mathrm{~cm}^{-1}$ in an FTIR spectrophotometer (Perkin-Elmer 1600 series). All the samples were analyzed using a single reflection ATR system (MKII Golden Gate ${ }^{\mathrm{TM}}$, Specac) with a diamond crystal (angle of incidence $45^{\circ}$; active sampling area $0.8 \times 0.8 \mathrm{~mm}$; depth of penetration $20 \mu \mathrm{m})$.

\section{Degradation and water uptake of SPLA50 microparticles}

Measuring the weight loss of the microparticles assessed the degradation behavior, and the water uptake behavior measured by the capability of the microparticles to incorporate water in their matrix (swelling), both parameters being studied over a period of 90 days. For this, SPLA50 microparticles were weighted and immersed in a phosphatebuffer saline (PBS) solution at physiological $\mathrm{pH}(\mathrm{pH} 7.4)$ at a 
ratio of $1 \mathrm{mg} / 1 \mathrm{~mL}$, and placed into a water bath at $37^{\circ} \mathrm{C}$ with constant agitation (60 rpm).

After predetermined time periods (up to 30 days for water uptake, 90 days for weight loss), the vials containing the immersed microparticles were centrifuged at low speed for particle deposition and the supernatant was removed for further measurements of degradation products. The remaining wet microparticles were weighted for water uptake measurements. Water uptake was determined using the following equation ${ }^{38}$ :

$$
\% \text { Water uptake }=\left[\left(m_{\mathrm{w}}-m_{\mathrm{i}}\right) / m_{\mathrm{i}}\right] \times 100,
$$

where $m_{\mathrm{i}}$ is the initial weight and $m_{\mathrm{w}}$ is the wet weight of the sample. After each time period, the samples were dried at $37^{\circ} \mathrm{C}$ and the final mass of the samples weighted to determine the weight loss according to the following equation ${ }^{38}$ :

$$
\% \text { Weight loss }=\left[\left(m_{\mathrm{f}}-m_{\mathrm{i}}\right) / m_{\mathrm{i}}\right] \times 100,
$$

where $m_{\mathrm{f}}$ is the final weight of the dry sample and $m_{\mathrm{i}}$ is the initial weight of the sample. Triplicates were performed for each sample at each time period and the results are shown as mean \pm standard deviation.

\section{Determination of DEX, MP, and MPA loading efficiency and release profile}

\section{Loading efficiency}

The loading efficiency for the different steroids was determined by measuring the amount of corticosteroid remaining in the reaction medium (unloaded) where the microparticles were synthesized. Aliquots of $1 \mathrm{~mL}$ of the reaction medium were taken and the amount of DEX, MP, and MPA present in each sample was measured using HPLC as described in the section HPLC Analysis. The results were obtained from three independent experiments run in duplicate with reproducible results and the mean value is reported.

The loading efficiency was calculated from the following formula:

$$
\text { Loading efficiency }(\%)=(\text { CSi }- \text { CSr }) / C S i \times 100 \%
$$

where CSi is the initial amount of corticosteroid to be loaded and CSr the amount of corticosteroid remaining in the reaction medium (unloaded), where microparticles were synthesized.

\section{In vitro release studies}

In vitro release studies were conducted in PBS, $0.1 \mathrm{M}, \mathrm{pH}$ 7.4. Preweighted corticosteroid-loaded microparticles were immersed in sterile PBS at a ratio of $5 \mathrm{mg} / \mathrm{mL}$ under mild agitating conditions, at $37^{\circ} \mathrm{C}$. At predetermined time points, PBS aliquots were sampled for further quantification of the released corticosteroid. The results were obtained from two independent experiments run in duplicate with reproducible results and the mean value is reported.

\section{HPLC analysis}

Samples were extracted three times with a mixture of hexane and ethyl acetate (1:1), to selectively remove the steroids. The extracts were combined and evaporated under a nitrogen flow. The residues were redissolved in methanol and subjected to analysis by HPLC.

The HPLC system consisted of an auto-injector Midas Injector Spark, and a Beckman System Gold Solvent Module 126 coupled to a Detector Module 166. The separation of the samples was performed in a PurospherStar RP-18e column (250 $\leq 4.5 \mathrm{~mm}, 5-\mu \mathrm{m}$ particle size; Merck, Germany), using methanol and water as solvents. The following gradient was used: 50\% methanol for $10 \mathrm{~min}, 30 \%$ methanol-70\% water for $25 \mathrm{~min}$. The flow rate was $1 \mathrm{~mL} / \mathrm{min}$ and the detection wavelength used was $254 \mathrm{~nm}$. Quantification of DEX, MP, and MPA was performed using the internal standard method using Triamcinolone (Lederle, New York, NY) as the reference compound.

\section{RESULTS AND DISCUSSION}

\section{Synthesis of starch-based microparticles with encapsulated DEX, MP, and MPA}

Figures 1 and 2 show representative scanning electron microscope images of the unloaded and corticosteroid-loaded microparticles, respectively. The synthesis method yielded corticosteroid-loaded microparticles with morphologies similar to unloaded microparticles (Fig. 1). Therefore, the entrapment of the steroids does not seem to produce significant changes in the morphology of the microparticles.

For both unloaded and loaded microparticles, two different surfaces can be found, namely, microparticles with a rougher surface [Fig. 2(A)] and microparticles with a smoother surface [Fig. 2(C)]. For the rougher-surface microparticles, a porous surface is ob-

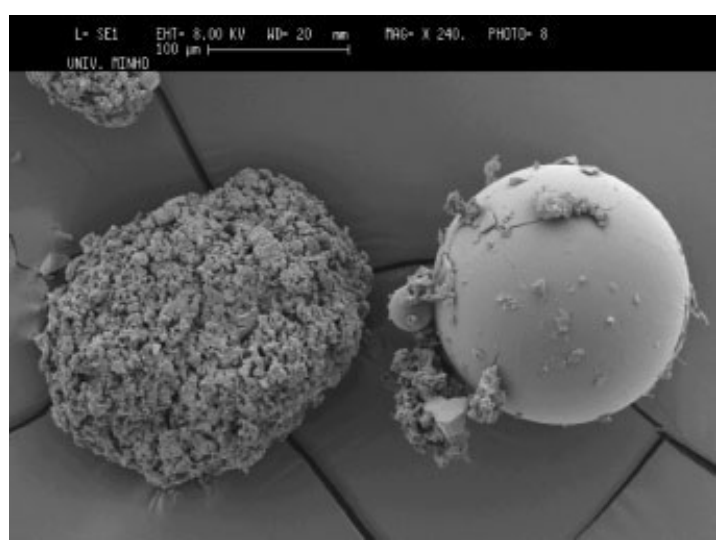

Figure 1. Representative image of unloaded SPLA50 microparticles showing the two different morphologies observed in each batch of microparticles, one displaying a rough surface and the other a smoother surface. 

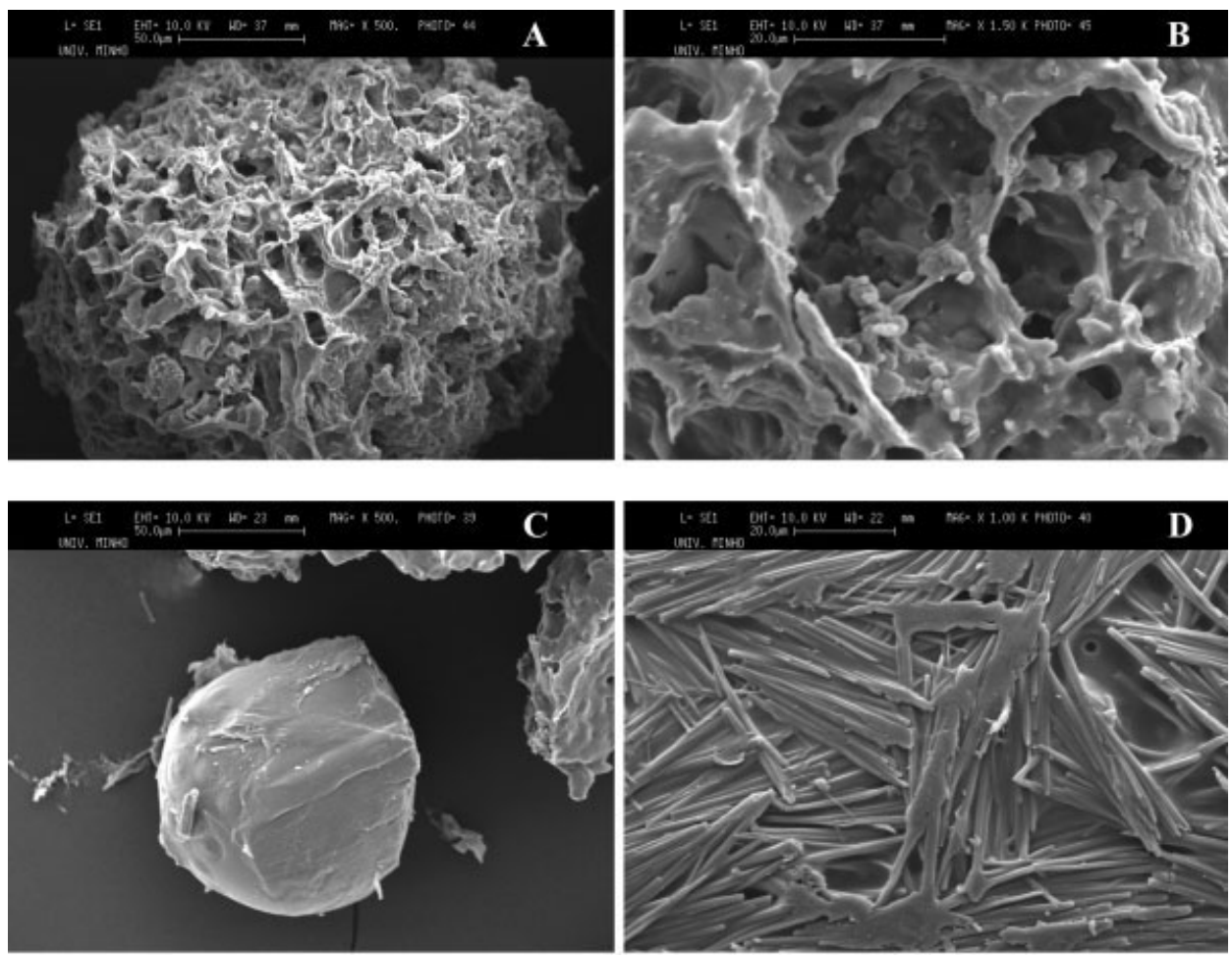

Figure 2. Morphology of the corticosteroid-loaded microparticles. In (A), the particle presents a rough surface, with apparent porosity [(B), detail of surface]; in (C) and (D), images of a microparticle displaying a smoother surface. Both morphologies are representative of the microparticles with different loaded corticosteroids.

servable at higher magnifications [Fig. 2(B)], which greatly enhances the surface area of the microparticle, compared with the surface of a smoother microparticle [Fig. 2(D)], in which no pores are visible.

Staining with iodine solution was performed to distinguish between starch and PLA phases, because iodine is known to bind to starch, yielding a dark blue color. Iodine staining revealed that the rough surface is due to the starch phase (stained as dark blue areas), and the smooth surface is due to the PLA phase (results not shown). This morphological feature, combined with the hydrophobic PLA and hydrophilic starch natures, can greatly influence the properties of the microparticles in terms of degradation and release profiles.

\section{Water uptake and degradation profiles of the microparticles}

The release of a drug from a matrix is primarily controlled by diffusion of the drug through the polymer due to its swelling in aqueous medium, and the dissolution rate of the drug, erosion of the polymer being an additional but nonetheless very important factor. ${ }^{39}$ For biodegradable polymers, it is essential to recognize that degradation is a chemical process, whereas erosion is a physical phenomenon dependent on dissolution and diffusion process. ${ }^{39}$ In this way, knowledge of the water uptake capability and degradation of polymeric materials is of great importance in studies regarding their use for controlled release applications. For SPLA50 microparticles, the hydration and weight loss profiles are shown in Figures 3 and 4, respectively.

The establishment of the water uptake profile might give insight into the release mechanism because, in the

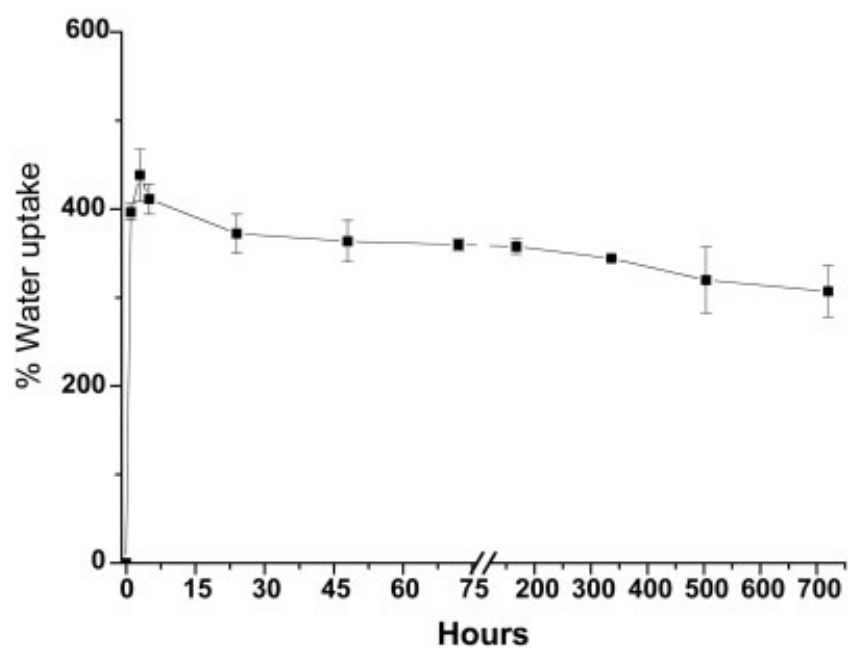

Figure 3. Water uptake profile of SPLA50 microparticles as determined by immersion in a PBS solution for up to 30 days $(n=9)$. 


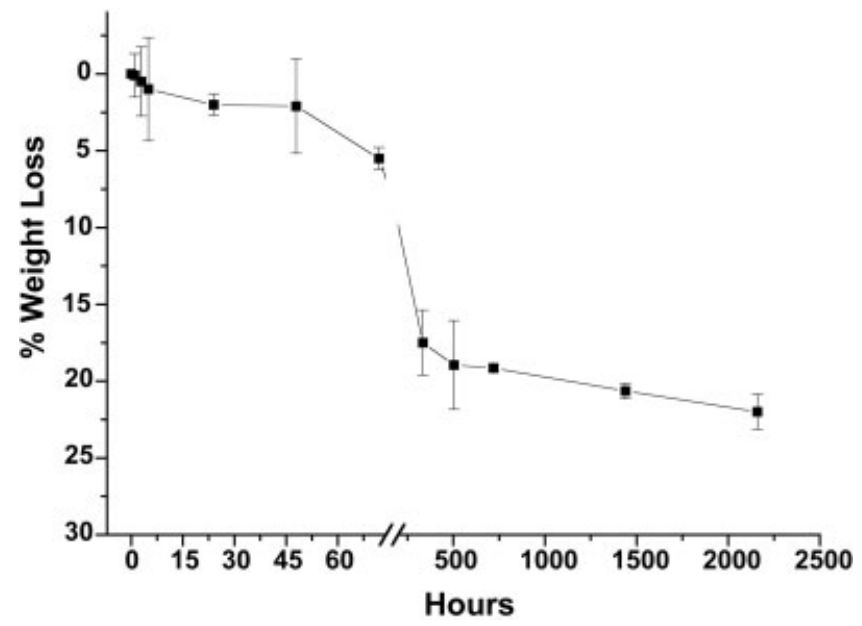

Figure 4. Weight loss profile of SPLA50 microparticles as determined by immersion in a PBS solution up to $2160 \mathrm{~h}$ (90 days) $(n=9)$.

case of polymers, drug diffusion is primarily controlled by swelling of the polymer matrix. In the present case, SPLA50 microparticles have the ability to swell to values as high as $400 \%$ of its weight, after $12 \mathrm{~h}$ of soaking, reaching equilibrium after 1 week in PBS solution, with a value around $300 \%$ (Fig. 3). After the initial release stage controlled by swelling of the matrix, subsequent release is primarily controlled by degradation of the polymer (chemical process). ${ }^{31}$ As so, monitoring the weight loss up to 90 days assessed the degradation of SPLA50 microparticles.

The weight loss profile of the microparticles in Figure 4 can be described by a reduced initial weight loss until $48 \mathrm{~h}$, followed by a period spanning from 48 to $500 \mathrm{~h}$ during which about $20 \%$ of weight is lost. Then a reduced decrease in weight is observed up to 90 days.

The first step in the degradation of the microparticles occurs mainly through scission of small-size starch chains into oligosaccharides, as assessed by the appearance of reducing sugars in solution (data not shown), up to $500 \mathrm{~h}$. The rate of degradation then decreases, because the cleavage of larger starch chains is to occur, this being coupled to the eventually slower degradation of the PLA phase. Further details on the degradation mechanisms of starch-based biomaterials can be found elsewhere. ${ }^{40-42}$

\section{Entrapment efficiency}

The entrapment/loading efficiency was determined by measuring the amount of corticosteroid remaining on the reaction media after the synthesis of the corticosteroid-loaded microparticles. Using HPLC to analyze aliquots of the reaction media (where corticoste- roid-loaded microparticles were synthesized), it was possible to quantify for unloaded corticosteroid. This value was then subtracted from the initial amount of corticosteroid to be loaded, enabling the efficiency of drug loading to be obtained (through the formula described in the section Loading Efficiency in Materials and Methods).

To optimize the loading, we studied whether varying the percentage of drug to be encapsulated would lead to a variation in the loading efficiency, in particular for DEX. Values of 2.5, 5, and 10\% were entrapped in the microparticles and the loading efficiency was evaluated.

As shown in Figure 5, the higher the initial amount of drug to be entrapped, the higher the encapsulation efficiency. The highest encapsulation efficiency was attained when $10 \%$ wt drug/wt polymer was used, with a value of $82 \%$. This drug-to-polymer ratio was chosen for subsequent studies with all the corticosteroids.

Table I displays the results of the loading efficiencies for each corticosteroid entrapped in the starchbased microparticles.

From Table I it is clear that DEX and MPA have the highest encapsulation efficiencies, with 82 and $84 \%$, respectively. The less successful encapsulation was attained for MP, with $51 \%$ loaded into the microparticles.

The differences in the loading efficiencies might be explained by the structure of the corticosteroids used (Fig. 6). The basic structure is the same; the only difference among these molecules is their side groups, which in DEX is a fluoride atom covalently bonded,

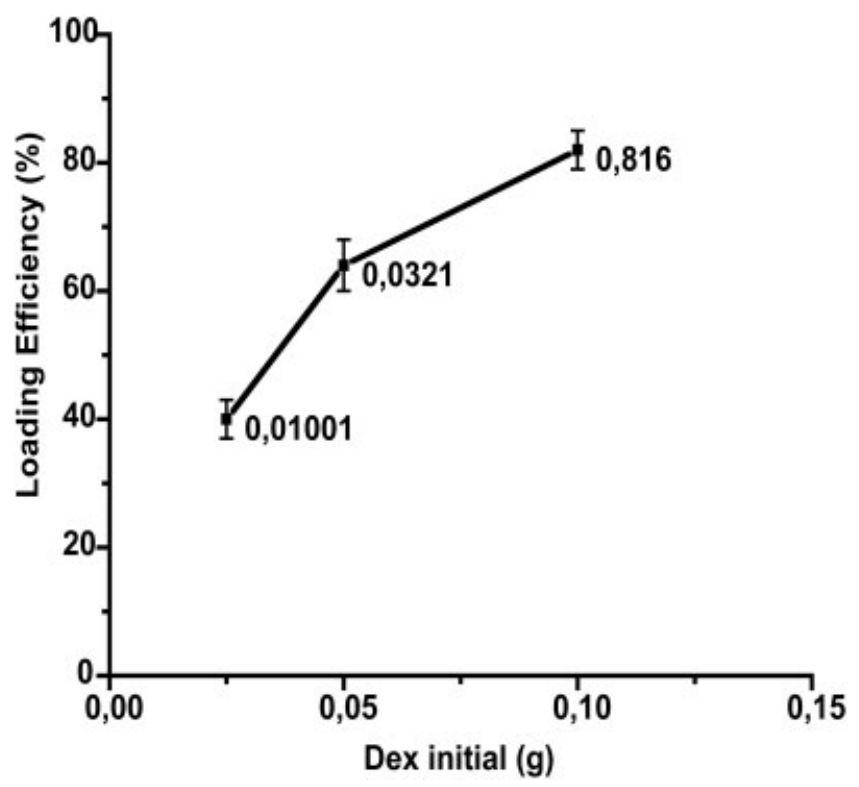

Figure 5. Influence of DEX initial amount of its loading efficiency. The amount (in grams) of loaded DEX is shown as labels. 
TABLE I

Loading Efficiencies for the Three Corticosteroids into the Starch-Based Microparticles at a Loading of $10 \% \mathrm{wt} /$ wt Drug/Polymer ${ }^{\text {a }}$

\begin{tabular}{lc}
\hline \multicolumn{1}{c}{ Corticosteroid } & $\begin{array}{c}\text { Loading } \\
\text { Efficiency (\%) }\end{array}$ \\
\hline Dexamethasone (DEX) & 82 \\
16 $\alpha$-Methylprednisolone (MP) & 51 \\
16 $\alpha$-Methylprednisolone acetate (MPA) & 84 \\
\hline
\end{tabular}

${ }^{a}$ Measuring the amount of corticosteroid remaining on the reaction media where the microparticles were formed and subtracting it from the initial amount of corticosteroid determined the efficiency of loading.

whereas for MPA is the acetate group. As for MP, it can be considered the basic structure when compared with the two other molecules.

MPA is a bulkier molecule than DEX or MP. This bulkiness could facilitate MPA entrapment within the polymer network that would result in the highest entrapment efficiency (Table I). This result correlates well with the data obtained from FTIR spectra, in which the presence of peaks due to drug loading is evident for DEX (Fig. 7) and also for MPA (Fig. 8), which can explain the high loading achieved. The entrapment efficiency is lower for MP (51\%, Table I), which by FTIR is shown by the absence of MP characteristic peaks in the spectrum (Fig. 9).

It is visible from the analysis of the FTIR spectra that for DEX (Fig. 7) and MPA (Fig. 8) there were some changes in the spectra because of drug loading in the SPLA50 microparticles, where bands characteristic of these corticosteroids appeared in the spectra of the loaded microparticles. This might be because some drug becomes trapped on/close to the surface of the polymer matrix during the manufacturing process, which is in accordance with descriptions in the literature for other systems. ${ }^{43,44}$ As for MP (Fig. 9), such changes were almost absent in the FTIR spectrum of the loaded microparticles, which as said before, can

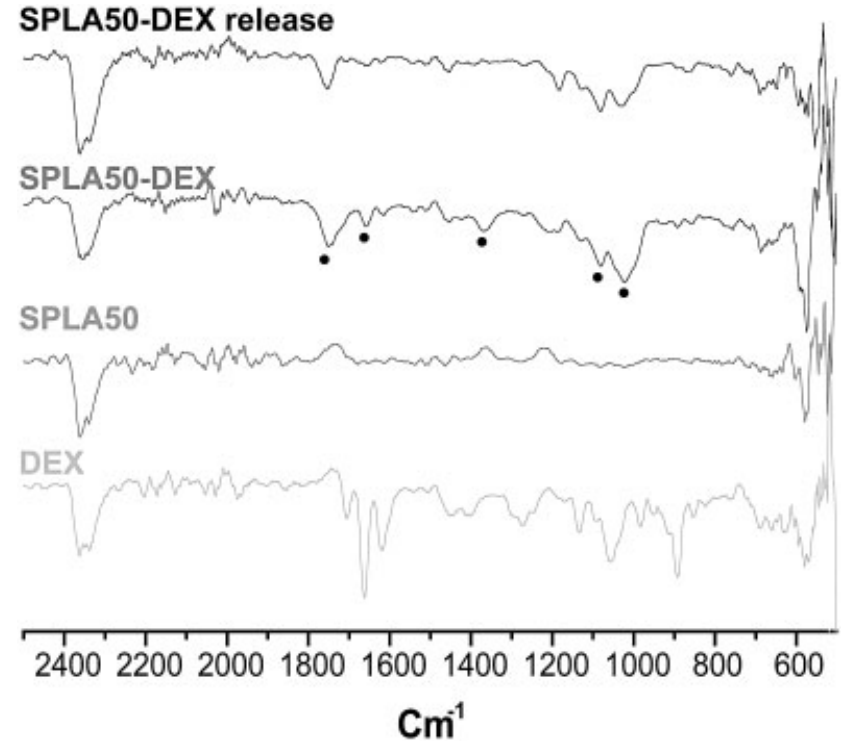

Figure 7. FTIR spectra of DEX, SPLA50, SPLA50-DEX loaded, and SPLA50-DEX released microparticles. The black dots (O) indicate bands present in the microparticles due to loading of DEX. After 30 days of release, the same bands are still visible, although a reduction in the intensity is observed.

explain the lower loading efficiency when compared with DEX and MPA.

For the MPA-loaded microparticles, there was a reduction in the intensity of the peaks because of the corticosteroid loading after 30 days of release, which seems to support the hypothesis that the corticosteroid trapped at the surface of the microparticles has been released at the initial stages of release. Regarding DEX, there was some reduction in the intensity of the bands because of corticosteroid loading but, nevertheless, they remain visible after 30 days of release.

In vitro release profile for $\mathrm{DEX}, \mathrm{MP}$, and $\mathrm{MPA}$

The in vitro release profiles for $\mathrm{DEX}(\mathrm{A}), \mathrm{MP}(\mathrm{B})$, and MPA (C) are shown in Figure 10. Analysis of the

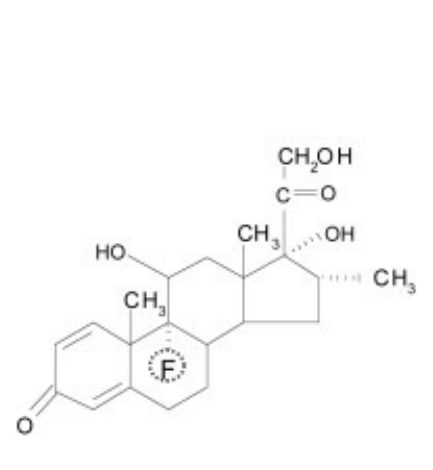

a)

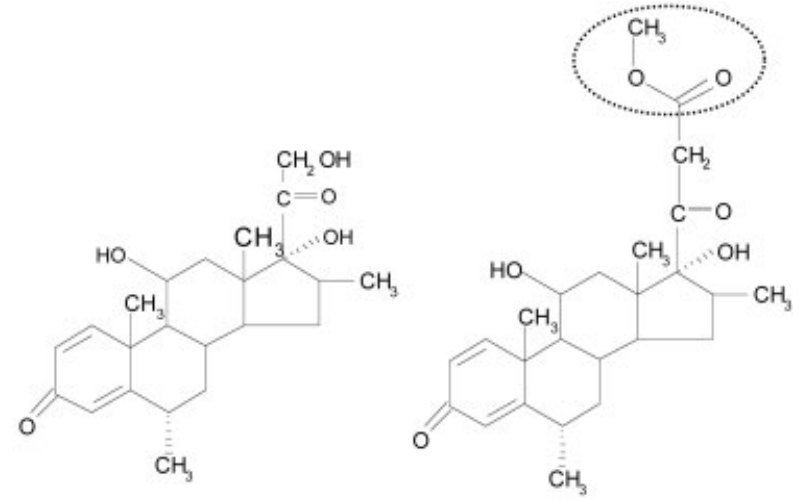

b) c)

Figure 6. Chemical structure for $\operatorname{DEX}(a), \operatorname{MP}(b)$, and MPA (c). 


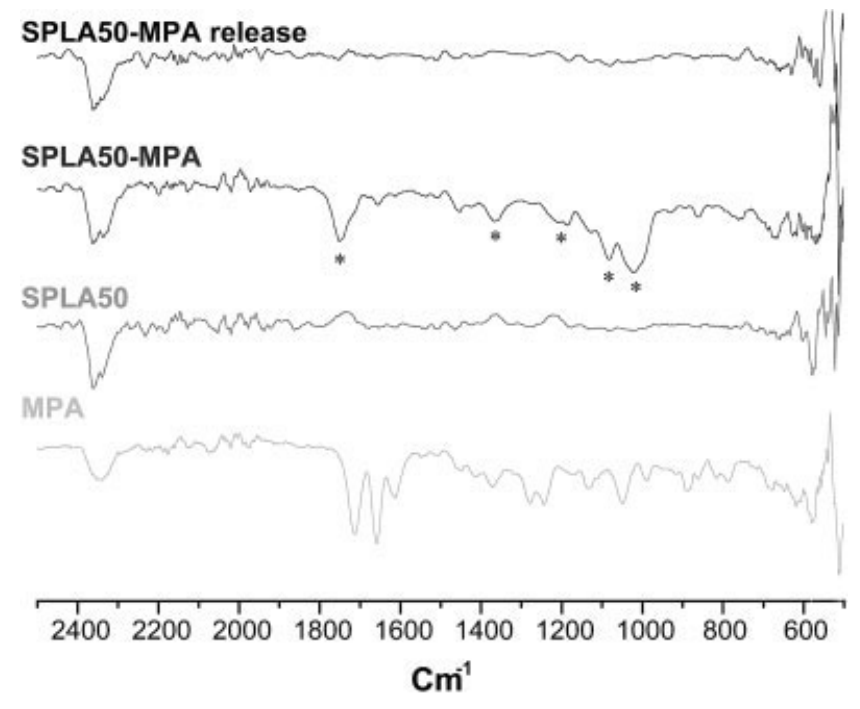

Figure 8. FTIR spectra of MPA, SPLA50, SPLA50-MPA loaded, and SPLA50-MPA released microparticles. The asterisks $\left({ }^{*}\right)$ indicate bands present in the microparticles due to loading with MPA. After release, these bands are almost absent from the structure of MPA-loaded microparticles.

release profiles indicates that all the corticosteroids are released with an initial burst phase, followed by a slower release, typical of a first order release kinetics. This is in accordance with what is described for other biodegradable polymers. ${ }^{43,45-50}$ The release profile is similar for DEX and MP, with 27 and $28 \%$ of release for the first $24 \mathrm{~h}$, respectively. This initial burst is then followed by a slower release until $750 \mathrm{~h}$ (30 days), in which $45 \%$ of entrapped DEX and $40 \%$ of MP are released. For MPA, a reduced burst effect is observed (approximately $12 \%$ for the first $24 \mathrm{~h}$ ) compared with the other two molecules. This initial release is then followed by a slower release that reaches $30 \%$ of the entrapped MPA by 30 days.

Interestingly, an incomplete release pattern is observed for all corticosteroids, which was also observed for chitin/poly(lactic-co-glycolic acid) 50:50 microspheres. ${ }^{51}$ This confirms the potential of this system for prolonged release of entrapped drugs.

The initial burst phase can be explained by the fact that the swelling of the material controls the initial release stages (Fig. 3, water uptake, first $12 \mathrm{~h}$ ), and the drug is thus released by diffusion through the channels created by water penetration. ${ }^{39,43,52}$ At this stage, degradation/weight loss does not have a significant role in release, because by $24 \mathrm{~h}$ only $2 \%$ of the weight is lost (Fig. 4, weight loss). The second, slower release period can be attributed to the weight loss, because this phenomenon is only evident after $48 \mathrm{~h}$, in which a decrease in weight of approximately $20 \%$ is observed until $500 \mathrm{~h}$.

The degradation and corticosteroid release of the microparticles after 30 days (750 h, Fig. 11) produce changes in the morphology of the microparticles, ev- idenced by pores in the surface of the microparticles. Nevertheless, the microparticle matrix structure is still maintained, which could account for the drug still remaining to be released.

The water uptake and weight loss do not account, however, for the differences observed among the corticosteroids. Comparing the release profiles of $\mathrm{MP}$ [Fig. 10(B)] and MPA [Fig. 10(C)], the differences in the release profile can be attributed to the structure of the molecule. Although MPA loading efficiency was higher than the one for MP (Table I), because the former has a more bulky structure than MP, its release was expected to be slower than the one for MP. Moreover, the presence of the acetate group in MPA [Fig. $6(\mathrm{C})$ ] renders to this molecule a more hydrophobic character than MP (considered the basic structure) [Fig. 6(B)] and thus MPA affinity toward the aqueous moiety is significantly reduced. This same explanation is applicable to the difference between DEX and MPA release: although they possess a similar loading efficiency (82 and $84 \%$, respectively), DEX was released faster and at a higher percentage than MPA, consistent with a molecule size effect.

When DEX is compared with MP, the difference in the molecule bulkiness [Fig. 6(A,B)] cannot explain the differences in the loading efficiencies. Furthermore, it does not seem to have any effect in the release profiles. Importantly, when all the release profiles are compared, it should be emphasized that the release into the medium is inversely correlated with increasing bulkiness and hydrophobicity of the molecules.

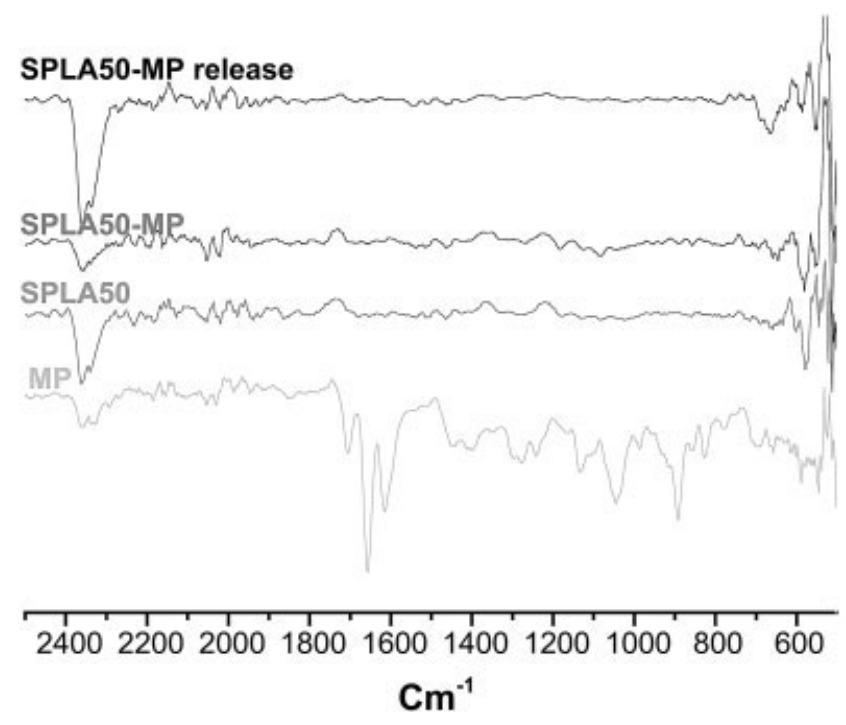

Figure 9. FTIR spectra of MP, SPLA50, SPLA50-MP loaded, and SPLA50-MP released microparticles. Microparticles loaded with MP show only minor changes in the structure compared with unloaded microparticles. The same observation is evident in the spectrum of microparticles after 30 days of MP release. 

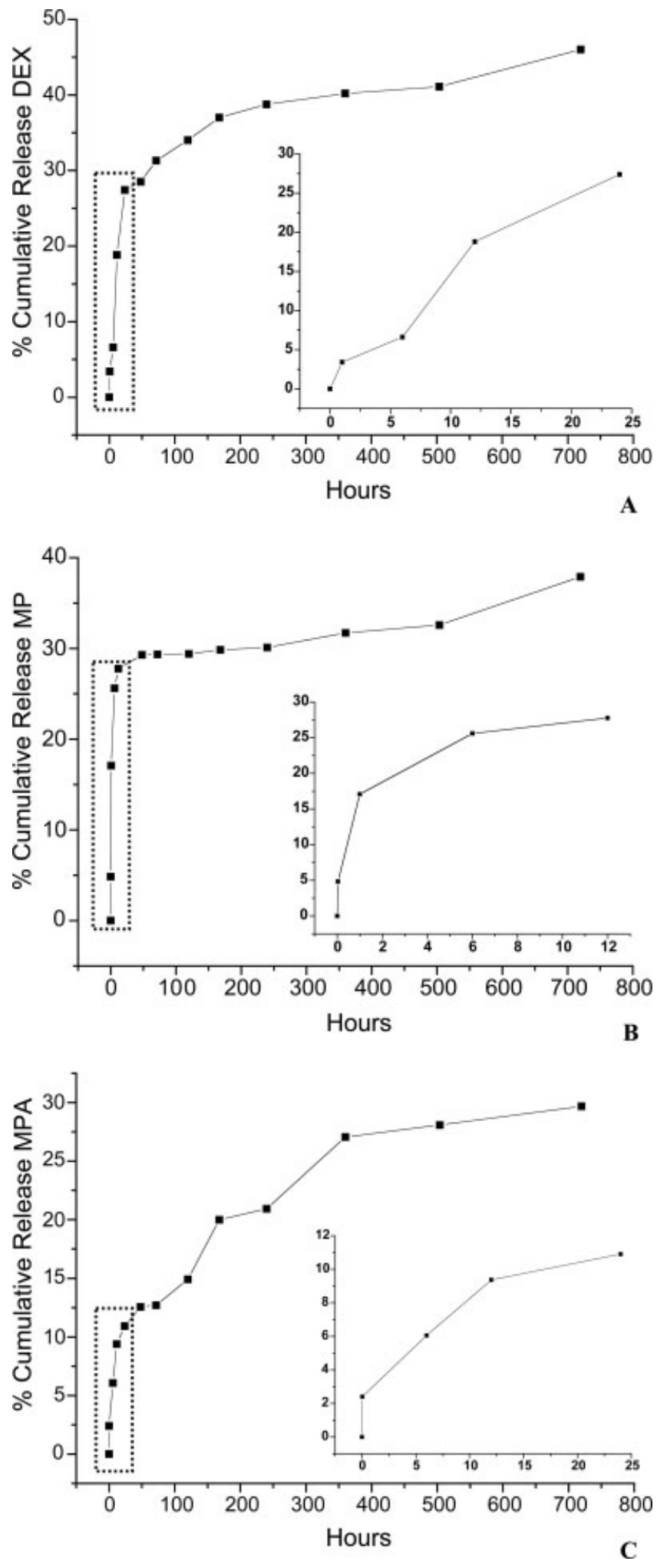

Figure 10. Release profiles as determined by HPLC quantification. (A) DEX, (B) MP, and (C) MPA. The results are shown as percentage of cumulative release. Right-insert graphs show the profile for the first $12[\mathrm{MP},(\mathrm{B})]$ and 24 [DEX, MPA, (A) and (C), respectively] h of release. A burst release occurs for all steroids, although for MPA this effect is less pronounced (12\%) compared with DEX (27\%) and MP $(28 \%)$.

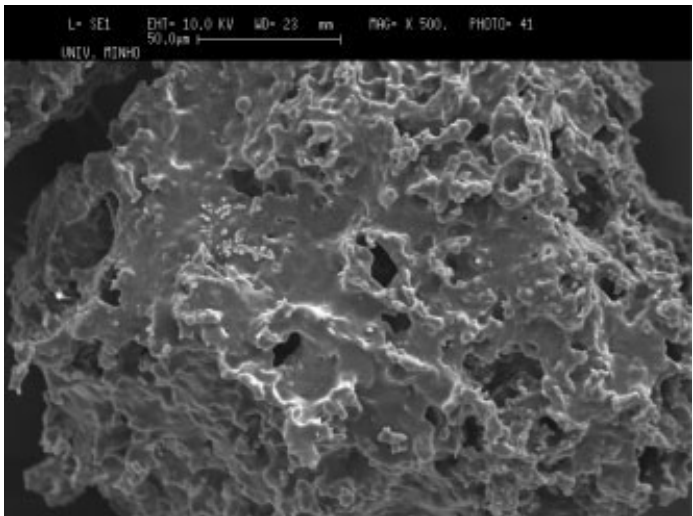

Figure 11. Representative images of the morphology of corticosteroid-loaded SPLA50 microparticles after 30 days of release in a PBS solution. Some pores are evident in the surface of the microparticles, but the matrix structure is still maintained.

\section{CONCLUSIONS}

In a previous work, ${ }^{30}$ by using a blend composed of starch and polylactic acid (SPLA50), we demonstrated the ability to form noncytotoxic microparticles within a defined size range. In this work, we evaluated the potential of these microparticles for release applications, by studying the loading and release of corticosteroids as model bioactive agents. We successfully encapsulated DEX and two other corticosteroids-MP and MPA - with the encapsulation efficiencies varying between 51 and $84 \%$. These differences might be explained by the structure of the molecules and the differential distribution of the molecule within the microparticle structure.

The in vitro release profiles showed that these starch-based systems are capable of sustained release of the entrapped steroids up to 30 days. The burst release observed in the first $24 \mathrm{~h}$ was lower for MPA when compared with DEX and MP. After this period, release proceeds for more than 30 days, with DEX and MP being released up to $45 \%$ and MPA up to $30 \%$. Whereas the water uptake can account for the initial burst release, degradation can account for the subsequent release stage. The remaining loaded corticosteroids could be released at later stages, and this release is likely controlled by the degradation of the microparticles.

From these studies we suggest that starch-based microparticles could be used as carriers for bioactive agents, and this investigation constitutes a basis for future entrapment and release studies using other bioactive agents, such as growth factors (bone morphogenetic proteins, vascular endothelial growth factor, platelet-derived growth factor) or others relevant for biomedical applications. 
G. A. Silva is the recipient of a scholarship from the Portuguese Foundation for Science and Technology.

\section{References}

1. Chellat F, Tabrizian M, Dumitriu S, Chornet E, Rivard $\mathrm{CH}$, Yahia L. Study of biodegradation behavior of chitosan-xanthan microspheres in simulated physiological media. J Biomed Mater Res 2000;53(5):592-599.

2. Reis RL, Cunha AM. Characterization of two biodegradable polymers of potential application within the biomaterials field. J Mater Sci Mater Med 1995;6(12):786-792.

3. Reis RL, Cunha AM, Allan PS, Bevis MJ. Mechanical behavior of injection-molded starch-based polymers. Polym Adv Technol 1996;7(10):784-790.

4. Reis RL, Mendes SC, Cunha AM, Bevis MJ. Processing and in vitro degradation of starch/EVOH thermoplastic blends. Polym Int 1997;43(4):347-352.

5. Reis RL, Cunha AM, Bevis MJ. Using nonconventional processing to develop anisotropic and biodegradable composites of starch-based thermoplastics reinforced with bone-like ceramics. Med Plast Biomater 1997;4:46-55.

6. Gomes ME, Ribeiro AS, Malafaya PB, Reis RL, Cunha AM. A new approach based on injection moulding to produce biodegradable starch-based polymeric scaffolds: Morphology, mechanical, and degradation behaviour. Biomaterials 2001;22(9): 883-889.

7. Mano JF, Korianova D, Reis RL. Thermal properties of thermoplastic starch/synthetic polymer blends with potential biomedical applicability. J Mater Sci Mater Med 2003;14:127-135.

8. Sousa RA, Mano JF, Reis RL, Cunha AM, Bevis MJ. Mechanical performance of starch based bioactive composite biomaterials molded with preferred orientation for potential medical applications. Polym Eng Sci 2002;42(5):1032-1045.

9. Sousa RA, Kalay G, Reis RL, Cunha AM, Bevis MJ. Injection molding of a starch/EVOH blend aimed as an alternative biomaterial for temporary applications. J Appl Polym Sci 2000; 77(6):1303-1315.

10. Sousa RA, Reis RL, Cunha AM, Bevis MJ. Structure and properties of hydroxylapatite reinforced starch bone-analogue composites. In: Proceedings from the Bioceramics 13 Meeting. 2000. p 669-672.

11. Sousa RA, Mano JF, Reis RL, Cunha AM, Bevis MJ. Mechanical performance of starch based bioactive composite biomaterials molded with preferred orientation. Polym Eng Sci 2002;42(5): 1032-1045.

12. Boesel LF, Mano JF, Elvira C, Reis RL. Hydrogels and hydrophilic partially degradable bone cements based on biodegradable blends incorporating starch. In: Chiellini E, Solaro R, editors. Biodegradable polymers and plastics. Dordrecht: Kluwer Academic; 2003.

13. Elvira C, Mano JF, San Roman J, Reis RL. Starch-based biodegradable hydrogels with potential biomedical applications as drug delivery systems. Biomaterials 2002;23(9):1955-1966.

14. Pereira CS, Cunha AM, Reis RL, Vazquez B, San Roman J. New starch-based thermoplastic hydrogels for use as bone cements or drug-delivery carriers. J Mater Sci Mater Med 1998;9(12): 825-833.

15. Malafaya PB, Elvira C, Gallardo A, San Roman J, Reis RL. Porous starch-based drug delivery systems processed by a microwave route. J Biomater Sci Polym Ed 2001;12(11):12271241.

16. Elvira C, Mano JF, San Roman J, Reis RL. Starch-based biodegradable hydrogels with potential biomedical applications as drug delivery systems. Biomaterials 2002;23(9):1955-1966.
17. Sousa RA, Reis RL, Cunha AM, Bevis MJ. Processing and properties of bone-analogue biodegradable and bioinert polymeric composites. Compos Sci Technol 2003;63(3-4):389-402.

18. Sousa RA, Oliveira AL, Reis RL, Cunha AM, Bevis MJ. Bi-composite sandwich moldings: Processing, mechanical performance, and bioactive behavior. J Mater Sci Mater Med 2003;14(5):385-397.

19. Gomes ME, Godinho JS, Tchalamov D, Cunha AM, Reis RL. Alternative tissue engineering scaffolds based on starch: Processing methodologies, morphology, degradation, and mechanical properties. Mater Sci Eng C 2002;20(1-2):19-26.

20. Mendes SC, Reis RL, Bovell YP, Cunha AM, van Blitterswijk CA, de Bruijn JD. Biocompatibility testing of novel starchbased materials with potential application in orthopaedic surgery: A preliminary study. Biomaterials 2001;22(14):2057-2064.

21. Gomes ME, Reis RL, Cunha AM, Blitterswijk CA, de Bruijn JD. Cytocompatibility and response of osteoblastic-like cells to starch-based polymers: Effect of several additives and processing conditions. Biomaterials 2001;22(13):1911-1917.

22. Salgado AJ, Gomes ME, Chou A, Coutinho OP, Reis RL, Hutmacher DW. Preliminary study on the adhesion and proliferation of human osteoblasts on starch-based scaffolds. Mater Sci Eng C 2002;20(1-2):27-33.

23. Marques AP, Reis RL, Hunt JA. The biocompatibility of novel starch-based polymers and composites: In vitro studies. Biomaterials 2002;23(6):1471-1478.

24. Gomes ME, Sikavitsas VI, Behravesh E, Reis RL, Mikos AG. Effect of flow perfusion on the osteogenic differentiation of bone marrow stromal cells cultured on starch-based threedimensional scaffolds. J Biomed Mater Res A 2003;67(1):87-95.

25. Mendes SC, Bezemer J, Claase MB, Grijpma DW, Bellia G, Degli-Innocenti F, Reis RL, de Groot K, van Blitterswijk CA, de Bruijn JD. Evaluation of two biodegradable polymeric systems as substrates for bone tissue engineering. Tissue Eng 2003; 9(Suppl 1):S91-S101.

26. Leonor IB, Reis RL. An innovative auto-catalytic deposition route to produce calcium-phosphate coatings on polymeric biomaterials. J Mater Sci Mater Med 2003;14(5):435-441.

27. Azevedo HS, Gama FM, Reis RL. In vitro assessment of the enzymatic degradation of several starch based biomaterials. Biomacromolecules 2003;4(6):1703-1712.

28. Oliveira AL, Elvira C, Reis RL, Vazquez B, San Roman J. Surface modification tailors the characteristics of biomimetic coatings nucleated on starch-based polymers. J Mater Sci Mater Med 1999;10(12):827-835.

29. Reis RL, Cunha AM, Fernandes MH, Correia RN. Treatments to induce the nucleation and growth of apatite-like layers on polymeric surfaces and foams. J Mater Sci Mater Med 1997; 8(12):897-905.

30. Silva GA, Costa FJ, Coutinho OP, Radin S, Ducheyne P, Reis RL. Synthesis and evaluation of novel bioactive starch/bioactive glass microparticles. J Biomed Mater Res 2004;70A:442-449.

31. Andrianov AK, Payne LG. Polymeric carriers for oral uptake of microparticulates. Adv Drug Delivery Rev 1998;34:155-170.

32. Langer R. Drug delivery systems. MRS Bull 1991;16(9):47-49.

33. Connolly AM, Schierbecker J, Renna R, Florence J. High dose weekly oral prednisone improves strength in boys with Duchenne muscular dystrophy. Neuromuscul Disord 2002;12:917-925.

34. Baeyens V, Kaltsatos V, Boisrame B, Varesio E, Veuthey J-L, Fathi M, Balant LP, Gex-Fabry M, Gurny R. Optimized release of dexamethasone and gentamicin from a soluble ocular insert for the treatment of external ophthalmic infections. J Controlled Release 1998;52(1-2):215-220.

35. Zignani M, Einmahl S, Baeyens V, Varesio E, Veuthey JL, Anderson J, Heller J, Tabatabay C, Gurny R. A poly(ortho ester) designed for combined ocular delivery of dexamethasone sodium phosphate and 5-fluorouracil: Subconjunctival tolerance and in vitro release. Eur J Pharm Biopharm 2000; 50(2):251-255. 
36. Lincoff AM, Furst JG, Ellis SG, Tuch RJ, Topol EJ. Sustained local delivery of dexamethasone by a novel intravascular eluting stent to prevent restenosis in the porcine coronary injury model. J Am Coll Cardiol 1997;29(4):808-816.

37. Maniatopoulos C, Sodek J, Melcher AH. Bone formation in vitro by stromal cells obtained from bone marrow of young adult rats. Cell Tissue Res 1988;254(2):317-330.

38. Demirgoz D, Elvira C, Mano JF, Cunha AM, Piskin E, Reis RL. Chemical modification of starch based biodegradable polymeric blends: Effects on water uptake, degradation behaviour and mechanical properties. Polym Degrad Stab 2000;70(2):161170.

39. Grassi M, Colombo I, Lapasin R. Drug release from an ensemble of swellable crosslinked polymer particles. J Controlled Release 2000;68(1):97-113.

40. Azevedo HS, Gama FM, Reis RL. In vitro assessment of the enzymatic degradation of several starch based biomaterials. Biomacromolecules 2003;4(6):1703-1712.

41. Araújo MA, Cunha AM, Mota M. Enzymatic degradation of starch based thermoplastic compounds used in prostheses. Mater Sci Forum 2004;455-456:429-432.

42. Vaz CM, Reis RL, Cunha AM. Degradation model of starchEVOH plus HA composites. Mater Res Innovations 2001;4(56):375-380.

43. Huang X, Brazel CS. On the importance and mechanisms of burst release in matrix-controlled drug delivery systems. J Controlled Release 2001;73:121-136.
44. Batycky RP, Hanes J, Langer R, Edwards DA. A theoretical model of erosion and macromolecular drug release from biodegradable microspheres. J Pharm Sci 1997;86:1464-1477.

45. Yoon JJ, Kim JH, Park TG. Dexamethasone-releasing biodegradable polymer scaffolds fabricated by a gas-foaming/saltleaching method. Biomaterials 2003;24(13):2323-2329.

46. Hanes J, Chiba M, Langer R. Degradation of porous poly(anhydride-co-imide) microspheres and implications for controlled macromolecule delivery. Biomaterials 1998;19:168-172.

47. Graham NB, McNeill ME. Hydrogels for controlled drug delivery. Biomaterials 1984;5(1):27-36.

48. Tuncel A, Cicek H, Hayran M, Piskin E. Monosize poly(ethylcyanoacrylate) microspheres: Preparation and degradation properties. J Biomed Mater Res 1995;29(6):721-728.

49. Vandamme TF, Lenourry A, Charrueau C, Chaumeil J-C. The use of polysaccharides to target drugs to the colon. Carbohydr Polym 2002;48(3):219-231.

50. Zignani M, Merkli A, Sintzel MB, Bernatchez SF, Kloeti W, Heller J, Tabatabay C, Gurny R. New generation of poly(ortho esters): Synthesis, characterization, kinetics, sterilization, and biocompatibility. J Controlled Release 1997;48(2-3):115-129.

51. Mi F-L, Shyu S-S, Lin Y-M, Wu Y-B, Peng C-K, Tsai Y-H. Chitin/PLGA blend microspheres as a biodegradable drug delivery system: A new delivery system for protein. Biomaterials 2003;24(27):5023-5036.

52. Soriano I, Llabres M, Evora C. Release control of albumin from polylactic acid microspheres. Int J Pharm 1995;125(2):223-230. 